\title{
AFFINE COHOMOLOGY CLASSES FOR FILIFORM LIE ALGEBRAS
}

\author{
DIETRICH BURDE
}

\begin{abstract}
We classify the cohomology spaces $H^{2}(\mathfrak{g}, K)$ for all filiform nilpotent Lie algebras of dimension $n \leq 11$ over $K$ and for certain classes of algebras of dimension $n \geq 12$. The result is applied to the determination of affine cohomology classes $[\omega] \in$ $H^{2}(\mathfrak{g}, K)$. We prove the general result that the existence of an affine cohomology class implies an affine structure of canonical type on $\mathfrak{g}$, hence a canonical left-invariant affine structure on the corresponding nilpotent Lie group. For certain filiform algebras the absence of an affine cohomology class implies the nonexistence of any affine structure. Of particular interest are algebras $\mathfrak{g}$ with minimal Betti numbers $b_{1}(\mathfrak{g})=b_{2}(\mathfrak{g})=2$.
\end{abstract}

\section{INTRODUCTION}

Left-invariant affine structures on Lie groups play an important role in the study of fundamental groups of compact affine manifolds and in the study of affine crystallographic groups. Milnor asked in his paper [11] on fundamental groups of complete affine manifolds whether or not every solvable Lie group admits a complete left-invariant affine structure. There was evidence that the answer should be positive. Auslander [2] had proved a converse statement: A Lie group admitting a complete left-invariant affine structure is solvable. Milnor's question became known as a conjecture. After a long history it was finally answered negatively. It was proved that there exist nilpotent Lie groups without any left-invariant affine structures, see [3],[5],[7]. All the known counterexamples are filiform nilpotent Lie groups of dimension $10 \leq n \leq 13$. The result also implies that there exist finitely generated torsionfree nilpotent groups which are not the fundamental group of any compact complete affine manifold. Moreover it follows that there exist nilmanifolds without any affine or projective structure. Besides the counterexamples there do not exist many results on Milnor's question. It is still unknown if there exist counterexamples in every dimension $n \geq 10$. The problem can be formulated in purely algebraic terms:

1.1. Definition. Let $A$ be a vector space over a field $K$ and let $A \times A \rightarrow A,(x, y) \mapsto x \cdot y$ be a $K$-bilinear product which satisfies

$$
x \cdot(y \cdot z)-(x \cdot y) \cdot z=y \cdot(x \cdot z)-(y \cdot x) \cdot z
$$

for all $x, y, z \in A$. Then $A$ together with the product is called left-symmetric algebra or LSA. The product is also called left-symmetric.

The term left-symmetric becomes evident, if we rewrite condition $(1)$ as $(x, y, z)=$ $(y, x, z)$, where $(x, y, z)=x \cdot(y \cdot z)-(x \cdot y) \cdot z$.

1.2. Definition. An affine structure or LSA-structure on a Lie algebra $\mathfrak{g}$ is a $K$-bilinear product $\mathfrak{g} \times \mathfrak{g} \rightarrow \mathfrak{g}$ which is left-symmetric and satisfies

$$
[x, y]=x \cdot y-y \cdot x
$$

1991 Mathematics Subject Classification. Primary 17B56, 17 B30. 
1.3. Proposition. Let $G$ be a simply connected Lie group with Lie algebra $\mathfrak{g}$. There is a canonical one-to-one correspondence between left-invariant affine structures on $G$ and affine structures on $\mathfrak{g}$.

The algebraic version of Milnor's question is the following: which Lie algebras over a field $K$ do admit affine structures. As mentioned above it is not quite true that all solvable Lie algebras admit an affine structure, although they tend to admit one. The case of reductive Lie algebras also is very interesting. There is a large literature on the subject, see for example [6] and the references cited within. Milnor's question is related to a refinement of Ado's theorem for finite-dimensional Lie algebras. If a Lie algebra $\mathfrak{g}$ of dimension $n$ admits an affine structure then $\mathfrak{g}$ possesses a faithful Lie algebra module of dimension $n+1$. The counterexamples to the existence of affine structures rely on the fact that not all nilpotent Lie algebras possess such a faithful module. It is however still very difficult to determine which nilpotent Lie algebras admit such modules. It requires a lot of computations to find out that just one particular nilpotent Lie algebra does not have a faithful module of small dimension. In general the question seems to be completely hopeless unless the Lie algebra $\mathfrak{g}$ is 2 -step, 3 -step or $n$-1-step nilpotent, where $n$ denotes the dimension of $\mathfrak{g}$. However, in the first two cases there always exist an affine structure and hence a faithful module of dimension $n+1$. The last case corresponds to filiform algebras. It is well known [1] that filiform algebras play also an interesting role in the study of Betti numbers $b_{i}(\mathfrak{g})$ of nilpotent Lie algebras $\mathfrak{g}$. They produce lower bounds for the Betti numbers. More precisely computations have shown that for any small $n$, there exists a filiform algebra $\mathfrak{f}_{n}$ such that $b_{i}\left(\mathfrak{f}_{n}\right) \leq b_{i}(\mathfrak{g})$ for all $i$ and all nilpotent Lie algebras $\mathfrak{g}$ of dimension $n$. Such filiform Lie algebras very often do not admit any affine structure. The first step is to study the cohomology spaces $H^{2}(\mathfrak{g}, K)$. We will compute the cohomology for all filiform Lie algebras of dimension $n \leq 11$ and for filiform Lie algebras of dimension $n \geq 12$ satisfying certain properties. Here the algebras $\mathfrak{g}$ of a certain subclass have minimal Betti numbers $b_{1}(\mathfrak{g})$ and $b_{2}(\mathfrak{g})$ equal to 2 . We conjecture that these algebras for $n \geq 13$ do not admit any affine structure. We have proved it for $n=13$ so far.

The present article contains some results without proofs, which will appear in an extended version.

\section{Filiform NiLPotent LiE ALGEBRAS}

In the study of nilpotent Lie algebras the filiform algebras play an important role.

2.1. Definition. Let $\mathfrak{n}$ be a Lie algebra over a field $K$. The lower central series $\left\{\mathfrak{n}^{k}\right\}$ of $\mathfrak{n}$ is defined by $\mathfrak{n}^{0}=\mathfrak{n}, \mathfrak{n}^{k}=\left[\mathfrak{n}^{k-1}, \mathfrak{n}\right], k \geq 1$. The integer $p$ is called nilindex of $\mathfrak{n}$ if $\mathfrak{n}^{p}=0$ and $\mathfrak{n}^{p-1} \neq 0$. In that case $\mathfrak{n}$ is called $p$-step nilpotent. A nilpotent Lie algebra $\mathfrak{n}$ of dimension $n$ and nilindex $p=n-1$ is called filiform.

2.2. Remark. If we denote the type of a nilpotent Lie algebra $\mathfrak{n}$ by $\left\{p_{1}, p_{2}, \ldots, p_{r}\right\}$ where $\operatorname{dim}\left(\mathfrak{n}^{i-1} / \mathfrak{n}^{i}\right)=p_{i}$ for all $i=1, \ldots, r$, then the filiform Lie algebras are just the algebras of type $\{2,1,1, \ldots, 1\}$. That explains the name "filiform" which means threadlike. Note that the center of a filiform Lie algebra is one-dimensional.

2.3. Example. Let $L=L(n)$ be the $n$-dimensional Lie algebra defined by

$$
\left[e_{1}, e_{i}\right]=e_{i+1}, i=2, \ldots, n-1
$$

where $\left(e_{1}, \ldots, e_{n}\right)$ is a basis of $L(n)$ and the undefined brackets are zero. This is called the standard graded filiform. 
It turns out to be useful to consider adapted bases for filiform Lie algebras. We need a few definitions:

2.4. Definition. Let $\mathcal{I}_{n}$ be an index set given by

$$
\begin{aligned}
& \mathcal{I}_{n}^{0}=\{(k, s) \in \mathbb{N} \times \mathbb{N} \mid 2 \leq k \leq[n / 2], 2 k+1 \leq s \leq n\}, \\
& \mathcal{I}_{n}= \begin{cases}\mathcal{I}_{n}^{0} & \text { if } n \text { is odd, } \\
\mathcal{I}_{n}^{0} \cup\left\{\left(\frac{n}{2}, n\right)\right\} & \text { if } n \text { is even. }\end{cases}
\end{aligned}
$$

Let $\left(e_{1}, \ldots, e_{n}\right)$ be a basis of $L(n)$ with $\left[e_{1}, e_{i}\right]=e_{i+1}$ for $2 \leq i \leq n-1$. For any element $(k, s) \in \mathcal{I}_{n}$ we can associate a 2 -cocycle $\psi_{k, s} \in Z^{2}(L, L)$ for the Lie algebra cohomology with coefficients in the adjoint module $L$ as follows:

$$
\begin{aligned}
\psi_{k, s}\left(e_{1} \wedge e_{i}\right) & =0, \\
\psi_{k, s}\left(e_{k} \wedge e_{k+1}\right) & =e_{s}
\end{aligned}
$$

for $1 \leq i \leq n, 2 \leq k \leq n-1$. Then the condition $\psi_{k, s} \in Z^{2}(L, L)$ for basis vectors $e_{1}, e_{i}, e_{j}$ with $2 \leq i, j$ is given by

$$
\left[e_{1}, \psi_{k, s}\left(e_{i} \wedge e_{j}\right)\right]=\psi_{k, s}\left(\left[e_{1}, e_{i}\right] \wedge e_{j}\right)+\psi_{k, s}\left(e_{i} \wedge\left[e_{1}, e_{j}\right]\right)
$$

and we obtain the following formula:

(3) $\psi_{k, s}\left(e_{i} \wedge e_{j}\right)= \begin{cases}(-1)^{k-i}\left(\begin{array}{c}j-k-1 \\ k-i\end{array}\right)\left(\operatorname{ad} e_{1}\right)^{(j-k-1)-(k-i)} e_{s} & \text { if } 2 \leq i \leq k<j \leq n \\ 0 & \text { otherwise. }\end{cases}$

The $\psi_{k, s}$ defined by $(3)$ in fact lie in $Z^{2}(L, L)$. The following result is due to Vergne [12], where $K=\mathbb{C}$ :

2.5. Lemma. Any n-dimensional filiform Lie algebra is isomorphic to a Lie algebra $L_{\psi}$ with basis $\left(e_{1}, \ldots, e_{n}\right)$ whose Lie brackets are given by

$$
\left[e_{i}, e_{j}\right]=\left[e_{i}, e_{j}\right]_{L}+\psi\left(e_{i} \wedge e_{j}\right), 1 \leq i, j \leq n .
$$

Here $\psi$ is a 2-cocycle which can be expressed by

$$
\psi=\sum_{(k, s) \in \mathcal{I}_{n}} \alpha_{k, s} \psi_{k, s}
$$

with $\alpha_{k, s} \in K$. The 2-cocycle $\psi \in Z^{2}(L, L)$ defines an infinitesimal deformation $L_{\psi}$ of $L$. 2.6. Definition. A basis $\left(e_{1}, \ldots, e_{n}\right)$ of an $n$-dimensional filiform Lie algebra is called adapted, if the brackets relative to this basis are given by (4) with a 2 -cocycle $\psi=$ $\sum_{(k, s) \in \mathcal{I}_{n}} \alpha_{k, s} \psi_{k, s}$.

Using Lemma 2.5 and (3) we obtain:

2.7. Lemma. All brackets of an $n$-dimensional filiform Lie algebra in an adapted basis $\left(e_{1}, \ldots, e_{n}\right)$ are determined by the brackets

$$
\begin{aligned}
{\left[e_{1}, e_{i}\right] } & =e_{i+1}, \quad i=2, \ldots, n-1 \\
{\left[e_{k}, e_{k+1}\right] } & =\alpha_{k, 2 k+1} e_{2 k+1}+\ldots+\alpha_{k, n} e_{n}, \quad 2 \leq k \leq[(n-1) / 2] \\
{\left[e_{\frac{n}{2}}, e_{\frac{n+2}{2}}\right] } & =\alpha_{\frac{n}{2}, n} e_{n}, \quad \text { if } n \equiv 0(2)
\end{aligned}
$$


2.8. Lemma. The brackets of an n-dimensional filiform Lie algebra in an adapted basis are given by:

$$
\begin{aligned}
& {\left[e_{1}, e_{i}\right]=e_{i+1}, \quad i=2, \ldots, n-1} \\
& {\left[e_{i}, e_{j}\right]=\sum_{r=1}^{n}\left(\sum_{\ell=0}^{[(j-i-1) / 2]}(-1)^{\ell}\left(\begin{array}{c}
j-i-\ell-1 \\
\ell
\end{array}\right) \alpha_{i+\ell, r-j+i+2 \ell+1}\right) e_{r}, \quad 2 \leq i<j \leq n .}
\end{aligned}
$$

where the constants $\alpha_{k, s}$ are zero for all pairs $(k, s)$ not in $\mathcal{I}_{n}$.

Note that an adapted basis for a filiform Lie algebra is not unique. Nevertheless we can associate coefficients $\left\{\alpha_{k, s} \mid(k, s) \in \mathcal{I}_{n}\right\}$ to a filiform Lie algebra with respect to an adapted basis. We have $(n-3)^{2} / 4$ parameters if $n$ is odd, and $\left(n^{2}-6 n+12\right) / 4$ if $n$ is even. The Jacobi identity defines certain equations with polynomials in $K\left[\alpha_{k, s}\right]$. If $n<8$, there are no equations, i.e., the Jacobi identity is satisfied automatically. In general, with respect to an adapted basis, the polynomial equations are much simpler than usual. As an example, for filiform Lie algebras of dimension 9, the Jacobi identity with respect to $\left\{\alpha_{k, s} \mid(k, s) \in \mathcal{I}_{9}\right\}$ is given by the single equation $\alpha_{4,9}\left(2 \alpha_{2,5}+\alpha_{3,7}\right)-3 \alpha_{3,7}^{2}=0$.

2.9. Definition. Let $V$ be a vector space of dimension $n$ over an algebraically closed field $K$ of characteristic zero, with fixed basis $\left(e_{1}, \ldots, e_{n}\right)$. A Lie algebra structure on $V$ determines a multiplication table relative to the basis. If

$$
\left[e_{i}, e_{j}\right]=\sum_{k=1}^{n} c_{i j}^{k} e_{k}
$$

then the point $\left(c_{i j}^{k}\right) \in K^{n^{3}}$ is called a Lie algebra law.

The constants $c_{i j}^{k}$ are subject to algebraic equations given by the skew-symmetry and the Jacobi identity of the Lie bracket. They define a certain Zariski-closed set in $n^{3}-$ dimensional affine space with coordinates $c_{i j}^{k}, 1 \leq i, j, k \leq n$. The set of all Lie algebra laws is often called the variety of Lie algebra laws and is denoted by $\mathcal{L}_{n}(K)$.

2.10. Definition. Denote by $\mathcal{F}_{n}(K)$ the Zariski-open subset of $\mathcal{L}_{n}(K)$ defining $n$-dimensional filiform nilpotent Lie algebras over $K$. Let $\mathcal{A}_{n}(K)$ denote the subset of $\mathcal{F}_{n}(K)$ consisting of elements which are the structure constants of a filiform Lie algebra with respect to an adapted basis. If $\lambda \in \mathcal{F}_{n}(K)$ then we denote the corresponding Lie algebra by $\mathfrak{g}_{\lambda}$. Denote the class of $n$-dimensional filiform Lie algebras over $K$ by $\mathfrak{F}_{n}(K)$.

Lemma 2.5 implies:

2.11. Lemma. Let $\mathfrak{g} \in \mathfrak{F}_{n}(K)$. Then there exists a basis $\left(e_{1}, \ldots, e_{n}\right)$ such that the corresponding Lie algebra law belongs to $\mathcal{A}_{n}(K)$.

Investigating affine structures on filiform Lie algebras it turns out that the following subclasses are of importance. Let $\mathfrak{g}$ be a filiform Lie algebra of dimension $n \geq 7$ and $\mathfrak{g}^{1}=[\mathfrak{g}, \mathfrak{g}], \mathfrak{g}^{k}=\left[\mathfrak{g}^{k-1}, \mathfrak{g}\right]$ for $k \geq 2$. The following properties are isomorphism invariants of $\mathfrak{g}$ :

(a) $\mathfrak{g}$ contains a one-codimensional subspace $U \supseteq \mathfrak{g}^{1}$ such that $\left[U, \mathfrak{g}^{1}\right] \subseteq \mathfrak{g}^{4}$.

(b) $\mathfrak{g}$ contains no one-codimensional subspace $U \supseteq \mathfrak{g}^{1}$ such that $\left[U, \mathfrak{g}^{1}\right] \subseteq \mathfrak{g}^{4}$.

(c) $\mathfrak{g}^{\frac{n-4}{2}}$ is abelian, where $n$ is even.

(d) $\left[\mathfrak{g}^{1}, \mathfrak{g}^{1}\right] \subseteq \mathfrak{g}^{6}$. 
These properties can more naturally be formulated in terms of structure constants of an adapted basis.

2.12. Definition. Let $\mathfrak{A}_{n}^{1}(K)$ denote the class of filiform Lie algebras of dimension $n \geq 12$ satisfying properties (b),(c),(d). Let $\mathfrak{A}_{n}^{2}(K)$ denote the class of filiform Lie algebras of dimension $n \geq 12$ satisfying properties (b),(c), but not property (d).

These two classes are disjoint, in the sense that a Lie algebra from the first class cannot be isomorphic to one of the second class. The algebras of $\mathfrak{A}_{n}^{1}(K)$ and $\mathfrak{A}_{n}^{2}(K)$ have some remarkable properties concerning central extensions and affine structures.

\section{Affine cohomology Classes}

In this section we will prove that the existence of affine cohomology classes in $H^{2}(\mathfrak{g}, K)$ for filiform Lie algebras $\mathfrak{g}$ implies the existence of a canonical affine structure on $\mathfrak{g}$. It is then very interesting to study filiform Lie algebras with minimal second Betti number $b_{2}(\mathfrak{g})=2$. Such algebras do not admit an affine cohomology class and hence no affine structure of canonical type. However, in order to ensure that there exists no other affine structures one needs additional conditions.

Let us quickly review Lie algebra cohomology, for details see [10]. Denote by $\mathfrak{g}$ a Lie algebra over $K$. Denote by $M$ an $\mathfrak{g}$-module with action $\mathfrak{g} \times M \rightarrow M,(x, m) \mapsto x \bullet m$. The space of $p$-cochains is defined by

$$
C^{p}(\mathfrak{g}, M)= \begin{cases}\operatorname{Hom}_{K}\left(\Lambda^{p} \mathfrak{g}, M\right) & \text { if } p \geq 0 \\ 0 & \text { if } p<0\end{cases}
$$

The coboundary operators $d_{p}: C^{p}(\mathfrak{g}, M) \rightarrow C^{p+1}(\mathfrak{g}, M)$ are defined by

$$
\begin{aligned}
\left(d_{p} \omega\right)\left(x_{1} \wedge \cdots \wedge x_{p+1}\right)= & \sum_{1 \leq r<s \leq p+1}(-1)^{r+s} \omega\left(\left[x_{r}, x_{s}\right] \wedge x_{1} \wedge \cdots \wedge \widehat{x_{r}} \wedge \cdots \wedge \widehat{x_{s}} \wedge \cdots \wedge x_{p+1}\right) \\
& +\sum_{t=1}^{p+1}(-1)^{t+1} x_{t} \cdot \omega\left(x_{1} \wedge \cdots \wedge \widehat{x_{t}} \cdots \wedge x_{p+1}\right)
\end{aligned}
$$

for $p \geq 0$ and $\omega \in C^{p}(\mathfrak{g}, M)$. If $p<0$ then we set $d_{p}=0$. A standard computation shows $d_{p} \circ d_{p-1}=0$, hence the definition

$$
H^{p}(\mathfrak{g}, M)=\operatorname{ker} d_{p} / \operatorname{im} d_{p-1}=Z^{p}(\mathfrak{g}, M) / B^{p}(\mathfrak{g}, M)
$$

makes sense. This space is called the $p^{\text {th }}$ cohomology group of $\mathfrak{g}$ with coefficients in the $\mathfrak{g}$-module $M$. The elements from $Z^{p}(\mathfrak{g}, M)$ are called $p$-cocycles, and from $B^{p}(\mathfrak{g}, M)$ $p$-coboundaries. The sequence

$$
0 \rightarrow C^{0}(\mathfrak{g}, M) \stackrel{d_{0}}{\longrightarrow} C^{1}(\mathfrak{g}, M) \stackrel{d_{1}}{\longrightarrow} C^{2}(\mathfrak{g}, M) \rightarrow \cdots
$$

yields a cochain complex, which is called the standard cochain complex and is denoted by $\left\{C^{\bullet}(\mathfrak{g}, M), d\right\}$.

The space of 2-cocycles and 2-coboundaries is given explicitly as follows: 


$$
\begin{aligned}
Z^{2}(\mathfrak{g}, M)= & \left\{\omega \in \operatorname{Hom}\left(\Lambda^{2} \mathfrak{g}, M\right) \mid x_{1} \bullet \omega\left(x_{2} \wedge x_{3}\right)-x_{2} \bullet \omega\left(x_{1} \wedge x_{3}\right)+x_{3} \bullet \omega\left(x_{1} \wedge x_{2}\right)\right. \\
& \left.-\omega\left(\left[x_{1}, x_{2}\right] \wedge x_{3}\right)+\omega\left(\left[x_{1}, x_{3}\right] \wedge x_{2}\right)-\omega\left(\left[x_{2}, x_{3}\right] \wedge x_{1}\right)=0\right\} \\
B^{2}(\mathfrak{g}, M)= & \left\{\omega \in \operatorname{Hom}\left(\Lambda^{2} \mathfrak{g}, M\right) \mid \omega\left(x_{1} \wedge x_{2}\right)=x_{1} \bullet f\left(x_{2}\right)-x_{2} \bullet f\left(x_{1}\right)-f\left(\left[x_{1}, x_{2}\right]\right)\right. \\
& \quad \text { for some } f \in \operatorname{Hom}(\mathfrak{g}, M)\}
\end{aligned}
$$

There are important special cases of Lie algebra cohomology. If $M=K$ denotes the 1-dimensional trivial module, i.e., $x \bullet m=0$ for all $x \in \mathfrak{g}$, then the numbers $b_{p}(\mathfrak{g})=$ $\operatorname{dim} H^{p}(\mathfrak{g}, K)$ are of special interest. The number $b_{p}(\mathfrak{g})$ is called the $p^{\text {th }}$ Betti number. There are many questions regarding the Betti numbers of nilpotent Lie algebras. Among other things one would like to know good upper and lower bounds for each $b_{p}(\mathfrak{g})$. It is still an open conjecture whether or not the following is true for nilpotent Lie algebras:

$$
b_{2}(\mathfrak{g})>\frac{b_{1}(\mathfrak{g})^{2}}{4}
$$

This is called the $b_{2}$-conjecture. It is proved for algebras with $b_{1}(\mathfrak{g}) \leq 3$, for 2 -step nilpotent Lie algebras and for nilpotent Lie algebras $\mathfrak{g}$ with $\operatorname{dim} \mathfrak{g} / \mathfrak{z}(\mathfrak{g}) \leq 7$. For details see [9]. Another conjecture is the toral rank conjecture for nilpotent Lie algebras, stating

$$
\sum_{p=0}^{n} b_{p}(\mathfrak{g}) \geq 2^{\operatorname{dim} \mathfrak{z}(\mathfrak{g})}
$$

where $\mathfrak{z}(\mathfrak{g})$ denotes the center of $\mathfrak{g}$. That is also known only in few cases [8]. For filiform Lie algebras however both conjectures are clear. Nevertheless the explicit determination of Betti numbers of filiform algebras leads to formidable combinatorial problems, see [1].

We come now to the definition of an affine 2-cocycle:

3.1. Definition. Let $\mathfrak{g} \in \mathfrak{F}_{n}(K)$. A 2-cocycle $\omega \in Z^{2}(\mathfrak{g}, K)$ is called affine, if $\omega: \mathfrak{g} \wedge \mathfrak{g} \rightarrow$ $K$ is nonzero on $\mathfrak{z}(\mathfrak{g}) \wedge \mathfrak{g}$. A class $[\omega] \in H^{2}(\mathfrak{g}, K)$ is called affine if every representative is affine.

3.2. Lemma. Let $\mathfrak{g} \in \mathfrak{F}_{n}(K)$ and $\omega \in Z^{2}(\mathfrak{g}, K)$ be an affine 2 -cocycle. Then its cohomology class $[\omega] \in H^{2}(\mathfrak{g}, K)$ is affine and nonzero.

Proof. If $\mathfrak{z}(\mathfrak{g})=\operatorname{span}\{z\}$, then $\omega$ is affine iff $\omega(z \wedge y) \neq 0$ for some $y \in \mathfrak{g}$. For $\xi \in B^{2}(\mathfrak{g}, K)$ we have $\xi(z \wedge y)=f([z, y])=f(0)=0$ for some linear form $f \in \mathfrak{g}^{*}$. Hence $\omega$ is not a $2-$ coboundary and $[\omega]$ is affine.

Since the elements of $H^{2}(\mathfrak{g}, K)$ classify the equivalence classes of central extensions of $\mathfrak{g}$ by $K$ we obtain the following characterization:

3.3. Proposition. A Lie algebra $\mathfrak{g} \in \mathfrak{F}_{n}(K)$ has an extension

$$
0 \rightarrow \mathfrak{z}(\mathfrak{h}) \stackrel{\iota}{\rightarrow} \mathfrak{h} \stackrel{\pi}{\rightarrow} \mathfrak{g} \rightarrow 0
$$

with $\mathfrak{h} \in \mathfrak{F}_{n+1}(K)$ if and only if there exists an affine $[\omega] \in H^{2}(\mathfrak{g}, K)$.

Proof. The center $\mathfrak{z}(\mathfrak{g})=\operatorname{span}\{z\}$ is one-dimensional. Suppose that $\mathfrak{g}$ has such an extension. Then $\mathfrak{z}(\mathfrak{h})$ is a trivial $\mathfrak{g}$-module equal to $K$. The extension determines a unique class $[\omega] \in H^{2}(\mathfrak{g}, K)$ and we may assume that the Lie bracket is given by

$$
[(a, x),(b, y)]_{\mathfrak{h}}:=\left(\omega(x \wedge y),[x, y]_{\mathfrak{g}}\right)
$$


on the vector space $\mathfrak{h}:=K \oplus \mathfrak{g}$. Suppose that $\omega(z \wedge y)=0$ for all $y \in \mathfrak{g}$. Then $(a, 0)$ and $(a, z)$ are contained in $\mathfrak{z}(\mathfrak{h})$. This contradicts $\mathfrak{z}(\mathfrak{h}) \cong K$. Hence $\omega$ is affine.

Conversely an affine $[\omega] \in H^{2}(\mathfrak{g}, K)$ determines an extension

$$
0 \rightarrow K \stackrel{\iota}{\rightarrow} \mathfrak{h} \stackrel{\pi}{\rightarrow} \mathfrak{g} \rightarrow 0
$$

via the Lie bracket $(8)$ on $\mathfrak{h}:=K \oplus \mathfrak{g}$. Let $(a, x) \in \mathfrak{z}(\mathfrak{h})$. Then $x \in \mathfrak{z}(\mathfrak{g})$ and it follows that $x$ is a multiple of $z$. Since $\omega(z, y) \neq 0$ for some $y \in \mathfrak{g}$ it follows that $(a, z)$ is not in $\mathfrak{z}(\mathfrak{h})$. Hence $x=0, \mathfrak{z}(\mathfrak{h})$ is the trivial one-dimensional $\mathfrak{g}$-module $K$ and $\mathfrak{h} \in \mathfrak{F}_{n+1}(K)$.

There is the following result on the connection between affine cohomology classes and affine structures:

3.4. Proposition. Let $\mathfrak{g} \in \mathfrak{F}_{n}(K)$ and assume that there exists an affine cohomology class $[\omega] \in H^{2}(\mathfrak{g}, K)$. Then $\mathfrak{g}$ admits an affine structure.

The proposition is a corollary of the following theorem:

3.5. Theorem. Let $\mathfrak{g} \in \mathfrak{F}_{n}(K)$ and suppose that $\mathfrak{g}$ has an extension

$$
0 \rightarrow \mathfrak{a} \stackrel{\iota}{\rightarrow} \mathfrak{h} \stackrel{\pi}{\rightarrow} \mathfrak{g} \rightarrow 0
$$

with $\iota(\mathfrak{a})=\mathfrak{z}(\mathfrak{h})$. Then $\mathfrak{g}$ admits an affine structure.

Proof. The first step of the proof consists in showing that we may assume $\mathfrak{h} \in \mathfrak{F}_{n+1}(K)$. For this we refer the reader to the extended version of this article. Let $\left(f_{1}, \ldots, f_{n+1}\right)$ be an adapted basis for $\mathfrak{h}$ and let $e_{i}:=f_{i} \bmod \mathfrak{z}(\mathfrak{h})$ for $i=1, \ldots, n$. Then $\left(e_{1}, \ldots, e_{n}\right)$ is an adapted basis of $\mathfrak{g}$. Let $\mathfrak{h}_{3}=\operatorname{span}\left\{f_{3}, \ldots, f_{n+1}\right\}$ and $\mathfrak{g}_{2}=\operatorname{span}\left\{e_{2}, \ldots, e_{n}\right\}$. There is a uniquely determined linear map $\varphi: \mathfrak{g} \rightarrow \mathfrak{h}_{3}$ satisfying $\varphi(x)=\left[f_{1}, \bar{x}\right]_{\mathfrak{h}}$ for all $x \in \mathfrak{g}$ where $\bar{x} \in \mathfrak{h}$ is any element with $\pi(\bar{x})=x$. The restriction of $\varphi$ to $\mathfrak{g}_{2}$ is bijective since it is evidently injective. Denote its inverse by $\psi: \mathfrak{h}_{3} \rightarrow \mathfrak{g}_{2}$. Now set for all $x, y \in \mathfrak{g}$

$$
x \bullet y:=\psi\left([\bar{x}, \varphi(y)]_{\mathfrak{h}}\right)
$$

The formula is well defined since $[\bar{x}, \varphi(y)]_{\mathfrak{h}}=\left[\bar{x},\left[f_{1}, \bar{y}\right]\right] \in \mathfrak{h}_{3}$. We will show that it satisfies conditions (1) and (2) of Definition 1.2 and hence defines an affine structure on $\mathfrak{g}$ :

$$
\begin{aligned}
x \bullet y-y \bullet x & =\psi\left(\left[\bar{x},\left[f_{1}, \bar{y}\right]\right]-\left[\bar{y},\left[f_{1}, \bar{x}\right]\right]\right) \\
& =\psi\left(\left[\bar{y},\left[f_{1}, \bar{x}\right]\right]-\left[f_{1},[\bar{y}, \bar{x}]\right]-\left[\bar{y},\left[f_{1}, \bar{x}\right]\right]\right) \\
& \left.=\psi\left(\left[f_{1},[\bar{x}, \bar{y}]\right]\right)=\psi\left(\left[f_{1}, \overline{[x, y}\right]_{\mathfrak{g}}\right]\right)=\psi\left(\varphi\left([x, y]_{\mathfrak{g}}\right)\right) \\
& =[x, y]_{\mathfrak{g}}
\end{aligned}
$$

where the brackets are taken in $\mathfrak{h}$ if not otherwise denoted. Using the identity $\left[f_{1}, \overline{\psi(w)}\right]=$ $w$ for all $w \in \mathfrak{h}_{3}$ and again the Jacobi identity we obtain for all $x, y, z \in \mathfrak{g}$ :

$$
\begin{aligned}
x \bullet(y \bullet z)-y \bullet(x \bullet z) & =\psi([\bar{x},[\bar{y}, \varphi(z)]]-[\bar{y},[\bar{x}, \varphi(z)]]) \\
& =[x, y]_{\mathfrak{g}} \bullet z \\
& =(x \bullet y) \bullet z-(y \bullet x) \bullet z
\end{aligned}
$$




\section{Computation of $H^{2}(\mathfrak{g}, K)$}

In this section we will completely determine the cohomology groups $H^{2}(\mathfrak{g}, K)$ for all $\mathfrak{g} \in \mathfrak{F}_{n}(K)$ with $n \leq 11$. We will also give some results for algebras from the classes $\mathfrak{A}_{n}^{1}(K)$ and $\mathfrak{A}_{n}^{2}(K)$. The cohomology spaces give important information on $\mathfrak{g}$. In our case, we obtain a complete description of the existence of affine cohomology classes. Let $\left(e_{1}, \ldots, e_{n}\right)$ be an adapted basis for $\mathfrak{g}$ so that its Lie algebra law lies in $\mathcal{A}_{n}(K)$.

4.1. Lemma. Let $\omega \in \operatorname{Hom}\left(\Lambda^{2} \mathfrak{g}, K\right)$. Then $\omega$ is an affine 2 -cocycle iff $\omega\left(e_{1} \wedge e_{n}\right)$ or $\omega\left(e_{2} \wedge e_{n}\right)$ is nonzero.

Proof. By definition, $\omega$ is affine iff $\omega\left(e_{j} \wedge e_{n}\right) \neq 0$ for some $j \in\{1, \ldots, n\}$. The condition for $\omega$ to be a $2-$ cocycle is as follows:

$$
\omega\left(\left[e_{i}, e_{j}\right] \wedge e_{k}\right)-\omega\left(\left[e_{i}, e_{k}\right] \wedge e_{j}\right)+\omega\left(\left[e_{j}, e_{k}\right] \wedge e_{i}\right)=0 \quad \text { for } i<j<k
$$

Setting $i=1, k=n$ we obtain $\omega\left(e_{j} \wedge e_{n}\right)=0$ for $3 \leq j \leq n$.

4.2. Definition. Define $\omega_{\ell} \in \operatorname{Hom}\left(\Lambda^{2} \mathfrak{g}, K\right)$ by the nonzero values as follows:

$$
\omega_{\ell}\left(e_{k} \wedge e_{2 \ell+3-k}\right)=(-1)^{k} \quad \text { for } 1 \leq \ell \leq[(n-1) / 2], 2 \leq k \leq[(2 \ell+3) / 2]
$$

In the following we will mainly use $\omega_{1}, \ldots, \omega_{4}$. They are defined by

$$
\begin{aligned}
& \omega_{1}\left(e_{2} \wedge e_{3}\right)=1 \\
& \omega_{2}\left(e_{2} \wedge e_{5}\right)=1, \omega_{2}\left(e_{3} \wedge e_{4}\right)=-1 \\
& \omega_{3}\left(e_{2} \wedge e_{7}\right)=1, \omega_{3}\left(e_{3} \wedge e_{6}\right)=-1, \omega_{3}\left(e_{4} \wedge e_{5}\right)=1 \\
& \omega_{4}\left(e_{2} \wedge e_{9}\right)=1, \omega_{4}\left(e_{3} \wedge e_{8}\right)=-1, \omega_{4}\left(e_{4} \wedge e_{7}\right)=1, \omega_{4}\left(e_{5} \wedge e_{6}\right)=-1
\end{aligned}
$$

4.3. Lemma. We have $\omega_{1}, \omega_{2} \in Z^{2}(\mathfrak{g}, K)$, whereas $\omega_{\ell}, \ell \geq 3$ need not be 2 -cocycles. If $\ell<[(n-1) / 2]$, then $\omega_{\ell}$ cannot be an affine 2 -cocycle.

Proof. The first claim follows easily from equation (10). In the case $i=1, j=2$ it reduces to $\omega_{1}\left(e_{3} \wedge e_{k}\right)=\omega_{1}\left(e_{k+1} \wedge e_{2}\right)$ for $k \geq 3$. On the other hand we have $\omega_{\ell}\left(e_{i} \wedge e_{n}\right)=0,1 \leq$ $i \leq n$ for $\ell<[(n-1) / 2]$.

It is interesting to consider filiform Lie algebras with minimal second Betti number. It is not difficult to show the following result:

4.4. Proposition. Let $\mathfrak{g} \in \mathfrak{F}_{n}(K), n \geq 6$ be a filiform Lie algebra with $b_{2}(\mathfrak{g})=2$. Then there exists no affine $[\omega] \in H^{2}(\mathfrak{g}, K)$.

We come now to the computation of the cohomology. We have to divide the filiform algebras into several well defined classes. However, the number of classes should be as small as possible. Hence we do not use the classification results of filiform Lie algebras. We divide $\mathcal{A}_{n}(K), 6 \leq n \leq 11$ into the following subsets depending on certain equalities 
or inequalities of the structure constants. These subsets correspond to well defined classes of filiform Lie algebras:

\begin{tabular}{|c|c|}
\hline Class & Conditions \\
\hline $\mathcal{A}_{6,1}$ & $\alpha_{3,6} \neq 0$ \\
\hline $\mathcal{A}_{6,2}$ & $\alpha_{3,6}=0$ \\
\hline $\mathcal{A}_{7,1}$ & $2 \alpha_{2,5}+\alpha_{3,7} \neq 0$ \\
\hline $\mathcal{A}_{7,2}$ & $2 \alpha_{2,5}+\alpha_{3,7}=0$ \\
\hline $\mathcal{A}_{8,1}$ & $\alpha_{4,8} \neq 0,2 \alpha_{2,5}+\alpha_{3,7}=0$ \\
\hline $\mathcal{A}_{8,2}$ & $\alpha_{4,8}=0,2 \alpha_{2,5}+\alpha_{3,7} \neq 0$ \\
\hline $\mathcal{A}_{8,3}$ & $\alpha_{4,8}=0,2 \alpha_{2,5}+\alpha_{3,7}=0, \alpha_{2,5} \neq 0$ \\
\hline $\mathcal{A}_{8,4}$ & $\alpha_{2,5}=\alpha_{3,7}=\alpha_{4,8}=0$ \\
\hline $\mathcal{A}_{9,1}$ & $2 \alpha_{2,5}+\alpha_{3,7} \neq 0, \alpha_{3,7}^{2} \neq \alpha_{2,5}^{2}$ \\
\hline $\mathcal{A}_{9,2}$ & $2 \alpha_{2,5}+\alpha_{3,7} \neq 0, \alpha_{3,7}^{2}=\alpha_{2,5}^{2}$ \\
\hline $\mathcal{A}_{9,3}$ & $\alpha_{2,5}=\alpha_{3,7}=0, \alpha_{4,9} \neq 0, \alpha_{2,6}+\alpha_{3,8} \neq 0$ \\
\hline $\mathcal{A}_{9,4}$ & $\alpha_{2,5}=\alpha_{3,7}=0, \alpha_{4,9} \neq 0, \alpha_{2,6}+\alpha_{3,8}=0$ \\
\hline $\mathcal{A}_{9,5}$ & $\alpha_{2,5}=\alpha_{3,7}=\alpha_{4,9}=0,2 \alpha_{2,7}+\alpha_{3,9} \neq 0$ \\
\hline $\mathcal{A}_{9,6}$ & $\alpha_{2,5}=\alpha_{3,7}=\alpha_{4,9}=0,2 \alpha_{2,7}+\alpha_{3,9}=0$ \\
\hline $\mathcal{A}_{10,1}$ & $\alpha_{5,10} \neq 0,2 \alpha_{2,5}+\alpha_{3,7} \neq 0$ \\
\hline $\mathcal{A}_{10,2}$ & $\alpha_{5,10} \neq 0,2 \alpha_{2,5}+\alpha_{3,7}=0$ \\
\hline $\mathcal{A}_{10,3}$ & $\alpha_{5,10}=0,2 \alpha_{2,5}+\alpha_{3,7} \neq 0, \alpha_{3,7}^{2} \neq \alpha_{2,5}^{2}$ \\
\hline $\mathcal{A}_{10,4}$ & $\alpha_{5,10}=0,2 \alpha_{2,5}+\alpha_{3,7} \neq 0 \alpha_{3,7}^{2}=\alpha_{2,5}^{2}$ \\
\hline $\mathcal{A}_{10,5}$ & $\alpha_{5,10}=0,2 \alpha_{2,5}+\alpha_{3,7}=0, \alpha_{4,9} \neq 0, \alpha_{2,6}^{2}+2 \alpha_{2,7} \alpha_{4,9} \neq 0$ \\
\hline $\mathcal{A}_{10,6}$ & $\alpha_{5,10}=0,2 \alpha_{2,5}+\alpha_{3,7}=0, \alpha_{4,9} \neq 0, \alpha_{2,6}^{2}+2 \alpha_{2,7} \alpha_{4,9}=0$ \\
\hline $\mathcal{A}_{10,7}$ & $\alpha_{5,10}=0,2 \alpha_{2,5}+\alpha_{3,7}=0, \alpha_{4,9}=0,2 \alpha_{2,7}+\alpha_{3,9} \neq 0$ \\
\hline $\mathcal{A}_{10,8}$ & $\alpha_{5,10}=0,2 \alpha_{2,5}+\alpha_{3,7}=0, \alpha_{4,9}=0,2 \alpha_{2,7}+\alpha_{3,9}=0, \alpha \neq 0$ \\
\hline $\mathcal{A}_{10,9}$ & $\alpha_{5,10}=0,2 \alpha_{2,5}+\alpha_{3,7}=0, \alpha_{4,9}=0,2 \alpha_{2,7}+\alpha_{3,9}=0, \alpha=0$ \\
\hline $\mathcal{A}_{11,1}$ & $2 \alpha_{2,5}+\alpha_{3,7} \neq 0,10 \alpha_{3,7}-\alpha_{2,5} \neq 0, \beta \neq 0$ \\
\hline $\mathcal{A}_{11,2}$ & $2 \alpha_{2,5}+\alpha_{3,7} \neq 0,10 \alpha_{3,7}-\alpha_{2,5} \neq 0, \beta=0$ \\
\hline $\mathcal{A}_{11,3}$ & $2 \alpha_{2,5}+\alpha_{3,7} \neq 0,10 \alpha_{3,7}-\alpha_{2,5}=0$ \\
\hline $\mathcal{A}_{11,4}$ & $2 \alpha_{2,5}+\alpha_{3,7}=0, \alpha_{4,9} \neq 0$ \\
\hline $\mathcal{A}_{11,5}$ & $\alpha_{2,5}=\alpha_{3,7}=\alpha_{4,9}=0, \alpha_{5,11} \neq 0,4 \alpha_{4,10}+2 \alpha_{3,8}-3 \alpha_{2,6} \neq 0, \alpha \neq 0$ \\
\hline $\mathcal{A}_{11,6}$ & $\alpha_{2,5}=\alpha_{3,7}=\alpha_{4,9}=0, \alpha_{5,11} \neq 0,4 \alpha_{4,10}+2 \alpha_{3,8}-3 \alpha_{2,6} \neq 0, \alpha=0$ \\
\hline $\mathcal{A}_{11,7}$ & $\alpha_{2,5}=\alpha_{3,7}=\alpha_{4,9}=0, \alpha_{5,11} \neq 0,4 \alpha_{4,10}+2 \alpha_{3,8}-3 \alpha_{2,6}=0, \gamma \neq 0$ \\
\hline $\mathcal{A}_{11,8}$ & $\alpha_{2,5}=\alpha_{3,7}=\alpha_{4,9}=0, \alpha_{5,11} \neq 0,4 \alpha_{4,10}+2 \alpha_{3,8}-3 \alpha_{2,6}=0, \gamma=0, \delta \neq 0$ \\
\hline $\mathcal{A}_{11,9}$ & $\alpha_{2,5}=\alpha_{3,7}=\alpha_{4,9}=0, \alpha_{5,11} \neq 0,4 \alpha_{4,10}+2 \alpha_{3,8}-3 \alpha_{2,6}=0, \gamma=0, \delta=0$ \\
\hline $\mathcal{A}_{11,10}$ & $\alpha_{2,5}=\alpha_{3,7}=\alpha_{4,9}=\alpha_{5,11}=0$ \\
\hline
\end{tabular}

where

$$
\begin{aligned}
& \alpha=3 \alpha_{4,10}\left(\alpha_{2,6}+\alpha_{3,8}\right)-4 \alpha_{3,8}^{2} \\
& \beta=\left(2 \alpha_{2,5}^{2}-5 \alpha_{3,7}^{2}\right)\left(4 \alpha_{2,5}^{2}-4 \alpha_{2,5} \alpha_{3,7}+3 \alpha_{3,7}^{2}\right) \\
& \gamma=22 \alpha_{3,8}^{2}-3 \alpha_{2,6} \alpha_{3,8}-9 \alpha_{2,6}^{2} \\
& \delta=\alpha_{5,11}\left(4 \alpha_{3,10}+5 \alpha_{2,8}\right)-3 \alpha_{4,11}\left(\alpha_{2,6}+\alpha_{3,8}\right)+2 \alpha_{2,7}\left(3 \alpha_{2,6}-11 \alpha_{3,8}\right)
\end{aligned}
$$


The result of the computation is as follows:

4.5. Proposition. The following table shows the cohomology spaces $H^{2}\left(\mathfrak{g}_{\lambda}, K\right)$ for all $\lambda \in \mathcal{A}_{n}(K), 3 \leq n \leq 11$. The corresponding Lie algebras $\mathfrak{g}_{\lambda}$ admit an affine cohomology class as follows:

\begin{tabular}{|c|c|c|c|c|}
\hline $\operatorname{dim} \mathfrak{g}_{\lambda}$ & Class & $H^{2}\left(\mathfrak{g}_{\lambda}, K\right)$ & affine $\omega$ & $b_{2}\left(\mathfrak{g}_{\lambda}\right)$ \\
\hline \hline 3 & $\mathcal{A}_{3}$ & $\omega_{1}, \omega$ & $\checkmark$ & 2 \\
\hline 4 & $\mathcal{A}_{4}$ & $\omega_{1}, \omega$ & $\checkmark$ & 2 \\
\hline 5 & $\mathcal{A}_{5}$ & $\omega_{1}, \omega_{2}, \omega$ & $\checkmark$ & 3 \\
\hline 6 & $\mathcal{A}_{6,1}$ & $\omega_{1}, \omega_{2}$ & - & 2 \\
\hline 6 & $\mathcal{A}_{6,2}$ & $\omega_{1}, \omega_{2}, \omega$ & $\checkmark$ & 3 \\
\hline 7 & $\mathcal{A}_{7,1}$ & $\omega_{1}, \omega_{2}, \omega$ & $\checkmark$ & 3 \\
\hline 7 & $\mathcal{A}_{7,2}$ & $\omega_{1}, \omega_{2}, \omega_{3}, \omega$ & $\checkmark$ & 4 \\
\hline 8 & $\mathcal{A}_{8,1}$ & $\omega_{1}, \omega_{2}, \omega_{3}$ & - & 3 \\
\hline 8 & $\mathcal{A}_{8,2}$ & $\omega_{1}, \omega_{2}, \omega$ & $\checkmark$ & 3 \\
\hline 8 & $\mathcal{A}_{8,3}$ & $\omega_{1}, \omega_{2}, \omega_{3}$ & - & 3 \\
\hline 8 & $\mathcal{A}_{8,4}$ & $\omega_{1}, \omega_{2}, \omega_{3}, \omega$ & $\checkmark$ & 4 \\
\hline 9 & $\mathcal{A}_{9,1}$ & $\omega_{1}, \omega_{2}, \omega$ & $\checkmark$ & 3 \\
\hline 9 & $\mathcal{A}_{9,2}$ & $\omega_{1}, \omega_{2}, \omega, \omega^{\prime}$ & $\checkmark$ & 4 \\
\hline 9 & $\mathcal{A}_{9,3}$ & $\omega_{1}, \omega_{2}, \omega_{3}$ & - & 3 \\
\hline 9 & $\mathcal{A}_{9,4}$ & $\omega_{1}, \omega_{2}, \omega_{3}, \omega$ & $\checkmark$ & 4 \\
\hline 9 & $\mathcal{A}_{9,5}$ & $\omega_{1}, \omega_{2}, \omega_{3}, \omega$ & $\checkmark$ & 4 \\
\hline 9 & $\mathcal{A}_{9,6}$ & $\omega_{1}, \omega_{2}, \omega_{3}, \omega, \omega^{\prime}$ & $\checkmark$ & 5 \\
\hline 10 & $\mathcal{A}_{10,1}$ & $\omega_{1}, \omega_{2}, \omega_{3}$ & - & 3 \\
\hline 10 & $\mathcal{A}_{10,2}$ & $\omega_{1}, \omega_{2}, \omega_{3}, \omega_{4}$ & - & 4 \\
\hline 10 & $\mathcal{A}_{10,3}$ & $\omega_{1}, \omega_{2}, \omega$ & $\checkmark$ & 3 \\
\hline 10 & $\mathcal{A}_{10,4}$ & $\omega_{1}, \omega_{2}, \omega_{3}$ & - & 3 \\
\hline 10 & $\mathcal{A}_{10,5}$ & $\omega_{1}, \omega_{2}, \omega_{3}$ & - & 3 \\
\hline 10 & $\mathcal{A}_{10,6}$ & $\omega_{1}, \omega_{2}, \omega_{3}, \omega$ & $\checkmark$ & 4 \\
\hline 10 & $\mathcal{A}_{10,7}$ & $\omega_{1}, \omega_{2}, \omega_{3}, \omega$ & $\checkmark$ & 4 \\
\hline 10 & $\mathcal{A}_{10,8}$ & $\omega_{1}, \omega_{2}, \omega_{3}, \omega_{4}$ & - & 4 \\
\hline 10 & $\mathcal{A}_{10,9}$ & $\omega_{1}, \omega_{2}, \omega_{3}, \omega_{4}, \omega$ & $\checkmark$ & 5 \\
\hline 11 & $\mathcal{A}_{11,1}$ & $\omega_{1}, \omega_{2}$ & - & 2 \\
\hline 11 & $\mathcal{A}_{11,2}$ & $\omega_{1}, \omega_{2}, \omega$ & $\checkmark$ & 3 \\
\hline 11 & $\mathcal{A}_{11,3}$ & $\omega_{1}, \omega_{2}, \omega$ & $\checkmark$ & 3 \\
\hline 11 & $\mathcal{A}_{11,4}$ & $\omega_{1}, \omega_{2}, \omega_{3}$ & - & 3 \\
\hline 11 & $\mathcal{A}_{11,5}$ & $\omega_{1}, \omega_{2}, \omega_{3}$ & - & 3 \\
\hline 11 & $\mathcal{A}_{11,6}$ & $\omega_{1}, \omega_{2}, \omega_{3}, \omega_{4}$ & - & 4 \\
\hline 11 & $\mathcal{A}_{11,7}$ & $\omega_{1}, \omega_{2}, \omega_{3}, \omega$ & $\checkmark$ & 4 \\
\hline 11 & $\mathcal{A}_{11,8}$ & $\omega_{1}, \omega_{2}, \omega_{3}, \omega_{4}$ & - & 4 \\
\hline 11 & $\mathcal{A}_{11,9}$ & $\omega_{1}, \omega_{2}, \omega_{3}, \omega_{4}, \omega$ & $\checkmark$ & 5 \\
\hline & & & & \\
\hline
\end{tabular}

The notations here are as follows. By $\omega_{1}, \ldots, \omega_{4}$ we denote always the $2-$ cocycles defined by (11). The letter $\omega$ stands for an affine 2-cocycle, which might be different for distinct classes of Lie algebras. The same holds for $\omega^{\prime}$. For the cohomology spaces the table shows 
representing 2-cocycles for a basis. So $\omega_{1}, \omega_{2}, \omega$ in the table means that $\left(\left[\omega_{1}\right],\left[\omega_{2}\right],[\omega]\right)$ is a basis of $H^{2}\left(\mathfrak{g}_{\lambda}, K\right)$. Note that for $n \geq 5$ the two-dimensional subspace spanned by $\left[\omega_{1}\right],\left[\omega_{2}\right]$ is always contained in $H^{2}\left(\mathfrak{g}_{\lambda}, K\right)$. A checkmark denotes the existence and a minus sign the absence of an affine 2 -cocycle.

4.6. Remark. We have also determined the cohomology and the affine cohomology classes for the class $\mathcal{A}_{11,10}$. However, to state the result here would us require to introduce too many subclasses. On the other hand, it is not difficult to show that all such algebras admit an affine structure.

4.7. Remark. Let $\lambda \in \mathcal{A}_{6,1}$. Then $\mathfrak{g}_{\lambda}$ does not admit an affine $[\omega] \in H^{2}\left(\mathfrak{g}_{\lambda}, K\right)$. However, $\mathfrak{g}_{\lambda}$ admits an affine structure since there exists a nonsingular derivation.

The computations have been done with the computer algebra package REDUCE. For the Lie algebras of the classes $\mathfrak{A}_{n}^{1}(K)$ and $\mathfrak{A}_{n}^{2}(K)$ we have obtained the following results.

4.8. Theorem. Let $\mathfrak{g} \in \mathfrak{A}_{n}^{1}(K), n \geq 12$. Then $b_{2}(\mathfrak{g}) \geq 3$ and there always exists an affine cohomology class. Hence all algebras $\mathfrak{g} \in \mathfrak{A}_{n}^{1}(K), n \geq 12$ admit a canonical affine structure.

4.9. Proposition. Let $\mathfrak{g} \in \mathfrak{A}_{12}^{2}(K)$. Then $H^{2}(\mathfrak{g}, K)=\operatorname{span}\left\{\left[\omega_{1}\right],\left[\omega_{2}\right],[\omega]\right\}$, where $\omega$ is an affine 2-cocycle.

The last result generalizes to higher dimensions as follows: for $\mathfrak{g} \in \mathfrak{A}_{n}^{2}(K), n \geq 13$ the existence of an affine cohomology class depends on one certain polynomial condition $P_{n} \equiv 0$ in the structure constants of $\mathfrak{g}$ :

4.10. Theorem. Let $\mathfrak{g} \in \mathfrak{A}_{n}^{2}(K), n \geq 13$. Then

$$
H^{2}(\mathfrak{g}, K)= \begin{cases}\operatorname{span}\left\{\left[\omega_{1}\right],\left[\omega_{2}\right],[\omega]\right\} & \text { if } \mathfrak{g} \text { satisfies } P_{n} \equiv 0, \\ \operatorname{span}\left\{\left[\omega_{1}\right],\left[\omega_{2}\right]\right\} & \text { otherwise. }\end{cases}
$$

In particular, $b_{2}(\mathfrak{g})=2$ for all algebras $\mathfrak{g} \in \mathfrak{A}_{n}^{2}(K), n \geq 13$ not satisfying the polynomial condition $P_{n} \equiv 0$. These algebras have minimal second Betti numbers (among nilpotent Lie algebras) and are candidates for Lie algebras without affine structures. In that direction we could prove:

\subsection{Theorem. No Lie algebra $\mathfrak{g} \in \mathfrak{A}_{13}^{2}(K)$ admits any affine structure.}

More precisely we have proved that $\mu(\mathfrak{g}) \geq 15$, where $\mu(\mathfrak{g})$ denotes the minimal dimension of a faithful $\mathfrak{g}$-module. If $\mathfrak{g}$ admits an affine structure then it is not difficult to see that $\mu(\mathfrak{g}) \leq \operatorname{dim} \mathfrak{g}+1$. Hence the existence question of affine structures is connected to the question of a refinement of Ado's theorem. It is very difficult to compute the invariant $\mu(\mathfrak{g})$, in particular for nilpotent Lie algebras. Then the adjoint representation is not faithful. For abelian Lie algebras the invariant can be computed explicitly: denote by $\lceil x\rceil$ the ceiling of $x$, i.e., the least integer greater than or equal to $x$ and let $\mathfrak{g}$ be an abelian Lie algebra of dimension $n$ over an arbitrary field $K$. Then $\mu(\mathfrak{g})=\lceil 2 \sqrt{n-1}\rceil$. The nilpotent Lie algebras which are counterexamples to the Milnor conjecture satisfy $\mu(\mathfrak{g}) \geq \operatorname{dim} \mathfrak{g}+2$. For details see [4]. Returning to the cohomology $H^{2}(\mathfrak{g}, K)$ we think it is interesting to study the following problem:

Open problem. Does a Lie algebra $\mathfrak{g} \in \mathfrak{A}_{n}^{2}(K), n \geq 13$ satisfy $\mu(\mathfrak{g}) \geq n+2$ if and only if there is no affine $[\omega] \in H^{2}(\mathfrak{g}, K)$ ? 


\section{REFERENCES}

[1] G. F. Armstrong, S. Sigg. On the cohomology of a class of nilpotent Lie algebras. Bull. Austral. Math. Soc. 54 (1996), 517-527.

[2] L. Auslander: Simply transitive groups of affine motions. Am. J. of Math. 99 (1977), 809-826.

[3] Y. Benoist: Une nilvariété non affine. J. Diff. Geom. 41 (1995), 21-52.

[4] D. Burde: A refinement of Ado's Theorem. Archiv Math. 70 (1998), 118-127.

[5] D. Burde, F. Grunewald: Modules for certain Lie algebras of maximal class. J. Pure Appl. Algebra 99 (1995), 239-254.

[6] D. Burde: Left-invariant affine structures on reductive Lie groups. J. Algebra 181 (1996), 884-902.

[7] D. Burde: Affine structures on nilmanifolds. Int. J. of Math. 7 (1996), 599-616.

[8] G. Cairns, B. Jessup: New bounds on the Betti numbers of nilpotent Lie algebras. Comm. Algebra 25 (1997), 415-430.

[9] G. Cairns, B. Jessup, J. Pitkethly: On the Betti numbers of nilpotent Lie algebras of small dimension. Birkhäuser, Prog. Math. 145, (1997), 19-31.

[10] A. W. Knapp: Lie groups, Lie algebras, and cohomology. Math. Notes 34 (1988), Princeton University Press.

[11] J. Milnor: On fundamental groups of complete affinely flat manifolds. Advances in Math. 25 (1977), 178-187.

[12] M. Vergne: Cohomologie des algèbres de Lie nilpotentes. Application à l'étude de la variété des algèbres de Lie nilpotentes. Bull. Soc. Math. France 98 (1970), 81-116.

Mathematisches Institut, Heinrich-Heine-Universität, Universitätssstr. 1, 40225 DüsSELDORF, GERMANY

E-mail address: dietrich@math.uni-duesseldorf.de 